\title{
A Different Road Towards a Better Personality Science
}

\author{
Kate C. McLean \\ Western Washington University \\ Moin Syed \\ University of Minnesota
}

\begin{abstract}
We argue that a better personality science is one that is inclusive of diverse theories, methods, researchers, and participants. The road towards that science will involve serious reflective work on our history, biases, and the systemic inequities that we have built as personality scientists, which sustain the status quo. The suggestions proposed by Leising et al. systematically bias particular methods and tools, such as qualitative work, which we propose are necessary to better our science.
\end{abstract}

\section{Commentary}

There is no question that there is a need for a better personality science. Less clear, however, are the answers to what we think "better" actually means and how exactly we get there. At the 2021 conference of the Association for Research in Personality, all of the invited sessions focused on how to create a more diverse, equitable, and globally-minded personality science. The conference is largely focused on North American representation and perspectives, but there were other voices as well, and one of the invited sessions pertained to diversity and inclusion in personality research in Europe. Collectively, the sessions made plain that there is a hunger for change, and a need for change that, if not addressed, could derail the field. Attending that conference and reading the target article by Leising, Thielmann, Glöckner, Gärtner, and Schönbrodt (2021) left us with the sense that we have entered into two different understandings of reality.

Sessions at the conference focused heavily on reflecting on and improving our methods to generate a stronger knowledge base, just as Leising et al. did. The point of departure, however, is that improving our methods are not enough. Like many of our colleagues at the conference, we emphasize the need to understand how our methods are intertwined with the tremendous lack of inclusivity and diversity in our field, and how they reify existing power structures. Improving our methodological approach can not only lead to more robust conclusions, it can also make us think more deeply about what we do and how we do it. Perhaps most importantly, doing so will help us to understand and acknowledge the limits of what we do. This kind of humility and reflection is at the core of "steps towards a better personality science," though as we elaborate, this might be characterized as more of a sea change than steps.

Although we are fans of common ground and understand the need to speak the same language and share tools to work together, we found the emphasis on consensus decision-making much too strong and much too premature. First, as we elaborate below, we do not yet have the 
appropriate data or methodological development to make these decisions. Second, there was no proposal to integrate the necessary work of reflecting on the biases that would impact such decisions. Third, there was no discussion of who would be in the room to make these consensus decisions, or of the power dynamics intrinsic to our existing systems that are perpetuated with such practices. The risk of Leising et al.'s proposal is that decisions made by a select few will not only contain unacknowledged bias, but will also systematically marginalize and restrict the very kind of work that is needed to understand our own biases in measurement and the experiences and voices that have been left out of our science. The risk is the potential for no meaningful change at all.

This point is not hypothetical, as it is indeed already what we see in the field. The majority of our common tools have not been subject to sufficient tests of whether they are appropriate across diverse groups of people. In her talk at the conference, Monisha Pasupathi (2021) eloquently argued that if one takes diversity in the human condition seriously, we will need to understand that our common constructs, methods, and measures are not appropriate or relevant for all people. We are not physicists. We are not evolutionary biologists. We are scientists who study humans in all their messy complexities, which include their messy and complex cultural-historical contexts. We will have to realize the limits of what we are doing and do something different, which means we need to slow down and deepen our understanding, rather than prioritize rushed consensus based on limited knowledge.

The overarching problem is that the paper is targeted at the wrong level. Before we can make the large-scale movements towards the better science Leising et al. have envisioned, we need to have a much broader scope on where we have gone wrong - beyond the under-powered and unreplicated studies that have given rise to the current crisis. We need to understand the power dynamics and inequitable systems that supported and celebrated those scholars and programs of research that were based on faulty data and designs. We need to understand the historical context of why we value what we value (e.g., quantitative over qualitative; Syed, 2021a ). We need to understand how our biases and values as researchers shape why we study what we study (e.g., Dunn et al., 2021). We need to take a good hard look at what is missing in our science (Syed, 2021b). We need to do the work.

Engaging in this kind of reflection highlights how the current emphasis on "good science" comes from an entirely quantitative perspective. Descriptive and qualitative work are the very kinds of tools that will help us to understand the potential limits of our theories and measures, the appropriateness of our measures for different populations, and the variety in the human condition that we should be capturing if we are doing our job. Unfortunately, the scoring system proposed by Leising et al. places this kind of descriptive and qualitative work at an even greater disadvantage. Qualitative research - mentioned only very briefly in passing - would receive the very lowest of scores, not even scorable on multiple components of the system.

We also find it ironic that the authors argue against the quantity-focused form of assessment that dominates the field, instead advocating for an approach that is more nuanced and grounded in the actual practices of the field (even if incomplete). Perhaps the same 
appreciation of the bias of quantitative work, and the potential benefits of qualitative work, can be applied to our science.

In short, we don't offer tidy solutions because the first step is serious reflective work. We need to truly examine the science we have created, and let some of it go. Who knows what we might discover. 


\section{References}

Dunn, E. W., Chen, L., Proulx, J. D. E., Ehrlinger, J., \& Savalei, V. (2021). Can researchers' personal characteristics shape their statistical inferences? Personality and Social Psychology Bulletin, 47(6), 969-984. https://doi.org/10.1177/0146167220950522

Pasupathi, M. (2021). It's Past Time to Acknowledge that All Psychology is Cultural

Psychology. Paper presented at the Virtual Association for Research in Personality, July 2021.

Syed, M. (2021a). It's 2 x 2 designs all the way down: Social psychology's over-reliance on experiments needlessly restricts diversity in the field. Invited presentation at the Society for Personality and Social Psychology Annual Conference [online]. osf.io/gc3mu/

Syed, M. (2021b). Where are race, ethnicity, and culture in personality research? PsyArXiv. 10.31234/osf.io/m57ph 